\title{
Intersections
}

Canadian Journal of Music

Revue canadienne de musique

\section{Euler et les réseaux harmoniques}

\section{Franck Jedrzejewski}

Volume 29, numéro 2, 2009

URI : https://id.erudit.org/iderudit/1000040ar

DOI : https://doi.org/10.7202/1000040ar

Aller au sommaire du numéro

\section{Éditeur(s)}

Canadian University Music Society / Société de musique des universités canadiennes

ISSN

1911-0146 (imprimé)

1918-512X (numérique)

Découvrir la revue

Citer cet article

Jedrzejewski, F. (2009). Euler et les réseaux harmoniques. Intersections, 29(2), 66-83. https://doi.org/10.7202/1000040ar

\section{Résumé de l'article}

Dans cet article, on montre que le Tentamen de Leonhard Euler (1707-1783) apporte une solution arithmétique et musicale aux problèmes évoqués par Leibniz dans sa lettre à Christian Goldbach du 17 avril 1712, et que, contrairement à une idée largement répandue, les genres musicaux (genus musicum) ont été repris et développés par des théoriciens de la musique et des compositeurs. Tout d'abord par le physicien Adriaan Fokker (1887-1972) qui a sollicité de nombreux compositeurs hollandais, puis par l'école américaine qui, avec Harry Partch (1901-1974), Ervin Wilson (1928) et Ben Johnston (1926), a poursuivi le développement de ces théories dans l'exploration expérimentale de l'intonation juste et des univers non tempérés.
All Rights Reserved (C) Canadian University Music Society / Société de musique des universités canadiennes, 2010
Ce document est protégé par la loi sur le droit d'auteur. L'utilisation des services d’Érudit (y compris la reproduction) est assujettie à sa politique d'utilisation que vous pouvez consulter en ligne.

https://apropos.erudit.org/fr/usagers/politique-dutilisation/ 


\title{
EULER ET LES RÉSEAUX HARMONIQUES
}

\author{
Franck Jedrzejewski
}

\section{LES DEGRÉS DE SUAVITÉ}

Leonhard Euler est né quelques années après la publication du Musikalische Temperatur de Werckmeister, en 1691. Cette année marque l'instauration du tempérament égal dans lequel toutes les quintes ont le même rapport acoustique. On sait que le mathématicien Simon Stevin avait publié une table complète des fréquences de ce tempérament ${ }^{1}$ quelques années auparavant. Mais déjà vers 1563, on trouve des arguments contre les proportions mathématiques justes. Dans les lettres que Jean-Baptiste Benedetti (1530-1590) adresse à Cipriano de Rore, Benedetti prétend qu'il est impossible de chanter juste dans certains passages lorsqu'il faut maintenir des intervalles pythagoriciens ou zarliniens entre la basse et le soprano (Benedetti 1585, p. 280). Dans le passage invoqué, le soprano passe du ré au do dièse et revient au ré, alors que la basse commence sur un sol, passe au mi puis va vers la note la qui est tenue jusqu'à la fin du passage.

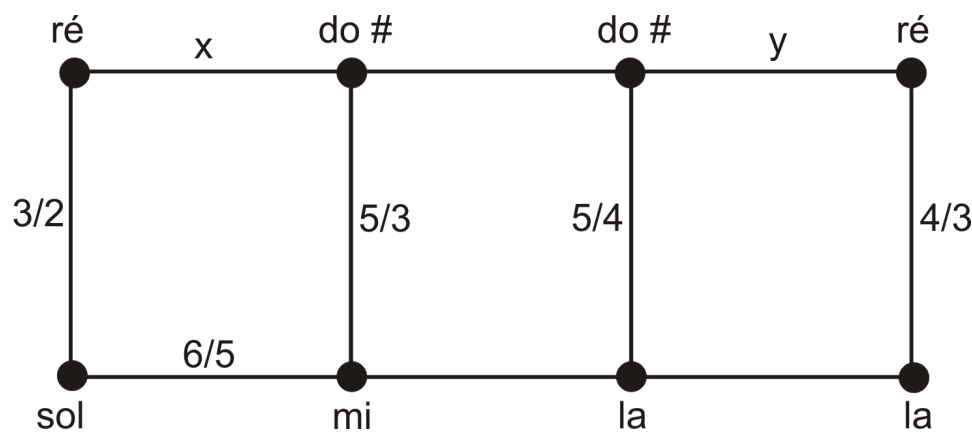

Figure 1.

Pour maintenir entre les autres notes les rapports de quinte juste $(3 / 2)$, de tierce mineure (6/5) et de tierce majeure (5/4) ou de leurs renversements, le soprano descend au ré dans un rapport $\mathrm{x}=27 / 25$ (solution de $(6 / 5) \cdot(3 / 2)=\mathrm{x}(5 / 3))$ et remonte au ré dans un rapport $\mathrm{y}=16 / 15$ (issu de l'équation $(4 / 3)=\mathrm{y}(5 / 4)$ ). La différence de ces deux rapports est un comma syntonique (81/80). Pour éviter ce

1 Les travaux de Stevin ont été édités par A. Fokker. S. Stevin, Van de Spiegheling der Singconst, dans Principal Works, ed. by Ernst Crone et al, Amsterdam : Swets \& Zeitlinger, 1985, vol. 5, 1966. 
comma disgracieux en harmonie tonale ( $1 / 8$ de ton environ), les musiciens sont contraints de chanter «faux » et ajustent naturellement à l'égal. Au demeurant, Benedetti est favorable au tempérament égal.

Euler, qui est donc né avec le tempérament égal, n’a que 24 ans, en 1731, lorsqu'il écrit le Tentamen novae theoriae musicae ex certissimis harmoniae principiis dilucide expositae (Essai d'une nouvelle théorie de la musique exposée en toute clarté selon les principes de l'harmonie les mieux fondés). L'œuvre écrite en latin ne sera publiée qu'en 1739. Euler y expose un mode de génération de systèmes acoustiques qu'il appelle genus musicum (Euler 1739, ch. 8 : De generibus musicis) et qui est traduit selon l'usage "genres d'Euler» ou " genres musicaux ». Rien ne prédisposait ce mathématicien à revenir sur les questions de l'accord des instruments, si ce n'est que ce fameux tempérament égal ne satisfaisait pas complètement les théoriciens et les compositeurs, et qu'il y avait une complicité naturelle entre les nombres et les fréquences du tempérament qui le fascinait. On reprochait au tempérament égal son caractère artificiel, qu’on opposait souvent au caractère naturel des fréquences en intonation juste. Mais pour Euler la perception des intervalles musicaux ne pouvait se faire quau travers de la perception des rapports numériques que les fréquences entretenaient entre elles. Leibniz le dit explicitement dans sa lettre du 17 avril 1712 à Christian Goldbach. Il voit dans la musique et les questions de consonance une pratique occulte de l'arithmétique.

Mais la raison des consonances doit être cherchée à partir de la congruence des chocs. La musique est un exercice occulte d'arithmétique de l'âme qui ignore qu'elle compte, car elle fait beaucoup de choses dans les perceptions confuses ou insensibles qu'elle ne peut remarquer par une aperception distincte. Car ceux qui croient qu'il ne peut rien y avoir en l'âme dont elle ne soit consciente se trompent. Donc, même si l'âme ne sent pas qu'elle compte, elle sent cependant l'effet de ce décompte insensible, c'està-dire dans le plaisir qui en résulte dans les consonances et dans le déplaisir des dissonances. (Leibniz 1988, p. 11)

C'est donc, pour Leibniz, la congruence des chocs qui détermine la consonance. Selon ce principe, l'oreille ne compte, dans une superposition de rythmes, que les frappes non simultanées. Dans la superposition d'un triolet et d'un duolet, le premier temps sera compté pour un coup et les suivants pour trois, formant ainsi quatre frappes (irrégulières) pour cette superposition. Ce principe s'étend à la perception des sons qui est considérée comme le résultat d'une série de chocs produite par l'onde acoustique sur le tympan. Cette idée est reprise par Euler dans le Tentamen (voir figure 2) et appliquée aux rapports acoustiques. Une note unique représente la pulsation élémentaire. C'est le niveau le plus simple de la perception. L'octave - de rapport $(2 / 1)-$ se justifie par la perception d'un choc tous les deux événements. Mais ce choc n'est pas nécessairement entendu : il reste dans le rapport du clair - confus de ce décompte insensible (i.e. qui se situe au-delà du sensible). L'octave représente le deuxième degré d'agrément de la perception des sons. Tous les autres intervalles fonctionnent de la même façon, mais à des degrés divers. Les congruences 


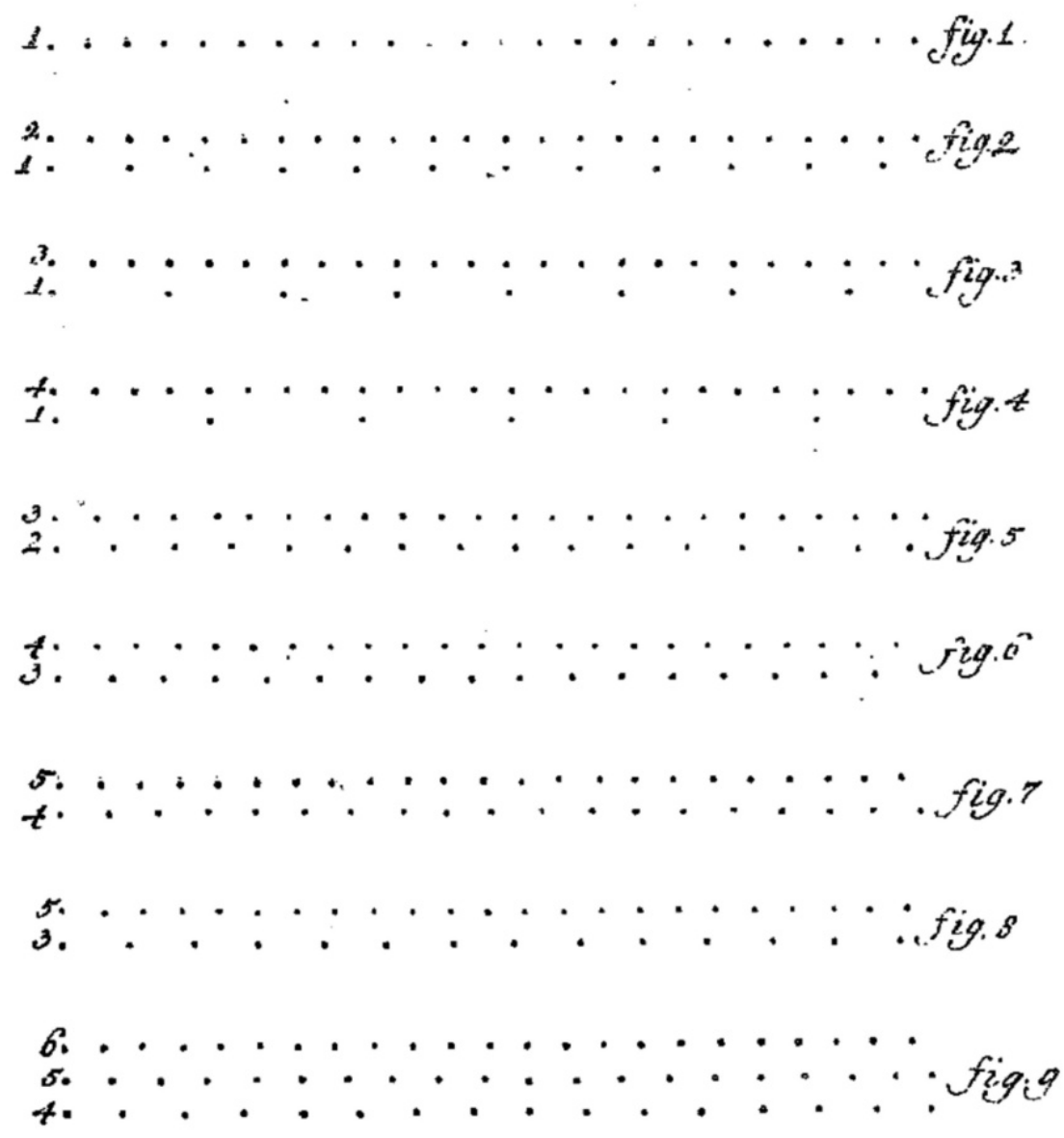

\section{T'ab. I}

Figure 2 : L. Euler, La congruence des chocs, Tentamen, 37.

sont de plus en plus complexes à mesure que les rapports des sons sont représentés par des nombres de plus en plus compliqués. La définition même de la congruence qu'en donne Leibniz justifie cette arithmétique occulte : «Sont congrues les choses qui, si elles sont différentes, ne peuvent être distinguées sans relation à quelque chose d'extérieur. » (Leibniz 1863, p. 29)

Non seulement le rapport acoustique se manifeste dans un son unique, mais même dans un accord, on retrouve toutes les composantes numériques des sons qui le composent. C'est le cas de la dernière ligne de l'exemple que nous offre Euler. L'accord parfait majeur composé d'une fondamentale (1), d'une tierce majeure $(5 / 4)$ et d'une quinte juste $(3 / 2)$ nous livre la succession des nombres 4:5:6 et la congruence des coups fournit une simultanéité de ces chocs tous les six événements. Plus cette simultanéité tardera à se produire, plus le son 
paraîtra dissonant. Ainsi, notre perception du son est liée à un principe virtuel de perception des nombres que la congruence des chocs explique en partie. Ce n'est pas du tout (comme on l'analysait au XIX siècle $^{2}$ ), une analogie visuelle ou un phénomène de la vue que l'on pourrait transposer à l'ouïe, mais bien une intellection auditive qui se fonde sur la correspondance d'un phénomène auditif et d'une relation numérique, quand bien même cette relation ne nous est pas explicitement donnée.

Euler donne de cette congruence des chocs une représentation numérique à travers le degré de suavité, en comptant d'abord la frappe simultanée du premier choc et en l'ôtant ensuite à chaque superposition d'événements non simultanés. Euler aurait pu proposer une autre fonction. Mais il aurait sans doute raté cette conjonction qu'il avait trouvée dans le degré de suavité reliant de manière profonde la théorie des nombres, la perception brute et son interprétation musicale. Pour mesurer la consonance ou la dissonance des sons, il part de son expression numérique. En termes modernes, Euler prend la décomposition en facteurs premiers du rapport acoustique $\mathrm{u}=\mathrm{p} / \mathrm{q}$ d'un son que nous écrivons sous la forme $\mathrm{u}=\mathrm{p}_{1}{ }^{\alpha_{1}} \ldots \mathrm{pn}^{\alpha \text { n }}$ où les entiers $\mathrm{p}_{1}, \ldots, \mathrm{pn}$ sont des nombres premiers et les exposants $\alpha_{1}, \ldots$, an des entiers relatifs, pour construire le degré de suavité (gradus suavitatis) qui est donné par la formule

$$
\mathrm{S}(\mathrm{u})=\sum_{k=1}^{n}\left|\alpha_{\mathrm{k}}\right|\left(\mathrm{p}_{\mathrm{k}}-1\right)
$$

Pour deux rapports, on établit facilement que le degré du produit est la somme des degrés diminuée d'une unité $\mathrm{S}(\mathrm{uv})=\mathrm{S}(\mathrm{u})+\mathrm{S}(\mathrm{v})-1$. Dans la table qui suit, Euler donne les rapports des principaux intervalles, ainsi que leur degré de suavité en chiffres romains (dernière colonne).

Toutefois, ce degré est contesté. Au lieu d'y voir un premier pas vers la difficile question de la distance harmonique, on condamne sans appel la notion. On lui reproche, tout d'abord, de ne pas correspondre à l'intuition de la consonance. La quarte (4/3) a un degré de suavité de 5, alors que la tierce majeure, considérée comme beaucoup plus agréable à l'audition que la quarte, a un degré de suavité de 7. Plus encore : la tierce mineure (6/5) a un degré de suavité de 8 , exactement comme le ton majeur (9/8) qui est considéré comme bien plus dissonant que la tierce mineure. Or, Euler avait prévu ces objections et voyait plus dans le degré de suavité un indicateur de la consonance qu'une réelle mesure de cette consonance : "À l'aide de notre méthode on pourra assigner jusqu'à un certain point, les limites qui séparent ces deux classes d'accords ; car les dissonances appartiennent aux degrés de suavité les plus élevés, et l'on considère comme des consonances les accords qui appartiennent aux degrés inférieurs. » (Euler 1739, p. 62 [Ch. 4, \$15])

Il n'y a pas de frontière qui sépare la consonance de la dissonance :

Si l'on examine la chose avec attention, dit Euler, on reconnaîtra que la différence des consonances et des dissonances ne consiste pas seulement

2 Voir par exemple Fétis 1840. 
dans la facilité de perception du rapport cherché, mais qu'elle doit être considérée dans l'ensemble de la composition du rapport. Les accords dont l'emploi est moins avantageux dans la musique sont appelés dissonances, alors même qu'ils sont plus faciles à apprécier que d'autres rangés parmi les consonances. (Euler 1739, p. $62[$ Ch. 4, \$ 15])

Euler est donc parfaitement informé des difficultés qu'il y a à vouloir partager les sons entre consonances et dissonances. La difficulté est encore plus grande lorsque l'on considère non plus des sons isolés mais des accords de sons. Pour déterminer le degré de suavité d'un accord formé de plusieurs sons, donc de plusieurs rapports, il faut d'abord réduire ses rapports en les multipliant par le plus grand commun diviseur des dénominateurs, puis en prendre le plus petit commun multiple pour définir ce que Euler appelle "l'exposant de l'accord » (Euler 1739, p. 66 [Ch. 4, \$ 23]). Le degré de suavité de l'accord est alors le degré de suavité de l'exposant de cet accord. Pour un accord parfait majeur (do, mi, sol) de rapports $(1,5 / 4,3 / 2)$, on déterminera dans un premier temps son plus grand diviseur 4 , qui donne l'expression de l'accord réduit $(4,5,6)$, puis, dans

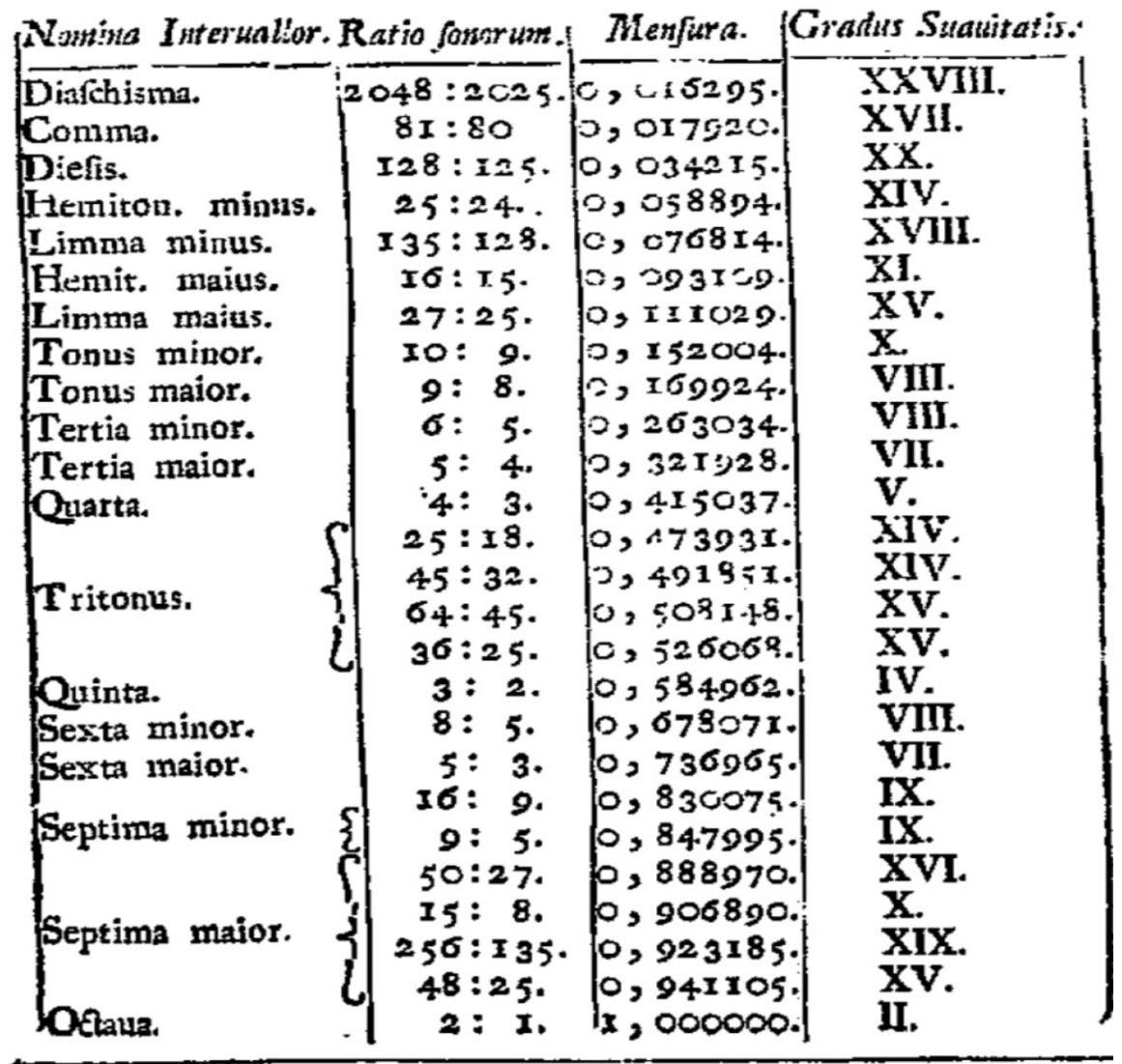

Figure $3:$ L. Euler, Degrés de suavité des intervalles, Tentamen, 112. 
un second temps, on prendra son plus petit commun multiple $u=4 \times 3 \times 5=60$ qui est l'exposant de l'accord. Le degré de suavité vaut alors 9 , ce qui est un degré de dissonance plus fort que celui de la seconde majeure.

Dans l'article de 1764, Euler revient sur la notion de dissonance et adopte des positions résolument modernes :

À cette occasion, il est important de remarquer que le mot dissonance est peu propre à exprimer l'idée qu'on y attache : cette idée n'est rien moins qu'opposée à celle qu'on attache au mot de consonance, comme l'étymologie semble l'indiquer ; et partant, puisque les consonances sont agréables à l'oreille, il ne faut pas s'imaginer que les dissonances lui soient désagréables, ou bien révoltantes : sur ce pied là, les dissonances devraient sans doute être entièrement bannies de toute la Musique. Les dissonances ne diffèrent donc des consonances proprement ainsi dites que parce qu'elles sont moins simples ou plus compliquées; et il est également nécessaire que cette plus grande complication soit aussi bien agréable à l'oreille, que la simplicité des consonances. (Euler 1766, p. 175)

\section{LES GENRES MUSICAUX}

À partir du chapitre VIII, Euler étudie ce qu'il appelle les genres de musique. Il ne s'agit pas de gammes comme on l'a parfois écrit, mais de systèmes acoustiques. Un système acoustique est un ensemble de fréquences ou de rapports de fréquences le plus souvent périodique à l'octave auquel on se réfère pour accorder les instruments. Une gamme est un ensemble de notes défini dans un même système acoustique, mais qui vaut pour plusieurs systèmes acoustiques. On parle de la gamme de do majeur dans le tempérament égal ou le tempérament pythagoricien pour exprimer une suite de notes (do, ré, mi fa, sol, la, si) indépendantes des fréquences que l'on attribue à ces notes.

Dans les genres d'Euler, on étudie avant tout les rapports numériques; ceuxci déterminent des rapports de fréquences, qui définissent des intervalles et par suite des successions de notes. Dans le Tentamen, Euler étudie les genres de la forme $2^{\mathrm{m}} 3^{\mathrm{n}} 5^{\mathrm{p}}$. Cette expression synthétique signifie que l'on considère comme appartenant à un même système acoustique tous les rapports de la forme $3^{i_{5}}{ }^{j}$ pour des entiers naturels $i=0,1, \ldots, n$ et $j=0,1, \ldots, p$, ces rapports étant recadrés dans l'intervalle de l'octave $[1,2]$ en multipliant ou en divisant par une puissance de 2 convenable (ce que signifie symboliquement le facteur $2^{\mathrm{m}}$, qui est en général omis dans les notations modernes). Euler obtient ainsi une table de 18 genres (Tentamen, 127-131). Les premiers genres n'ont que peu d'éléments : le genre I est formé d'un seul son de rapport 1 et de son octave 2. Le genre III est formé de trois sons $\{1,3 / 2,2\}$ : un son, sa quinte et son octave, de même que le genre $\mathrm{V}$ qui contient un son, sa tierce majeure et son octave $\{1,5 / 4,2\}$.

Les genres qui ne font intervenir que les puissances de 3 sont des systèmes pythagoriciens engendrés par les quintes justes $(3 / 2)$; ceux-ci n'intéressent pas Euler. Il remarque que certains genres sont inclus les uns dans les autres, certains sont trop simples pour avoir un intérêt musical, d'autres, au contraire, sont trop compliqués et convoquent des rapports inusités. Parmi les 
18 genres étudiés, Euler privilégie le genre $3^{3} 5^{2}$ qu'il appelle le genre diatonicochromatique $e^{3}$ et lui consacre le chapitre IX. Ce genre est la succession d'intervalles bien connus ${ }^{4}$ : le limma mineur $\ell=135 / 128$ de 92 cents, le limma majeur $\mathrm{L}=27 / 25$ de 133 cents, le demi-ton majeur $\mathrm{H}=16 / 15$ de 112 cents et le demi-ton mineur $\mathrm{h}=25 / 24$ de 71 cents. En notation moderne, ce genre s'exprime par le mot $\ell \mathrm{HhH} \ell \mathrm{HHhLhHH}$. Ce mot signifie que le premier intervalle est le limma mineur $\ell$, le deuxième le demi-ton majeur $\mathrm{H}$, etc. En général, Euler commence ses systèmes acoustiques sur la note $f a$, mais si l'on commence sur la note $d o$,

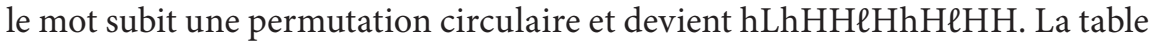
suivante décrit le genre en notation moderne.

\begin{tabular}{|l|c|c|c|c|c|}
\hline & Fréq. & Cents & Expr. & Longueurs & Intervalles \\
\hline C & 1 & 0 & $2^{7} \cdot 3$ & 384 & $\mathrm{~h}$ \\
\hline Cis & $25 / 24$ & 71 & $2^{4} \cdot 5^{2}$ & 400 & $\mathrm{~L}$ \\
\hline D & $9 / 8$ & 204 & $2^{4} \cdot 3^{3}$ & 432 & $\mathrm{~h}$ \\
\hline Dis & $75 / 64$ & 275 & $2 \cdot 3^{2} \cdot 5^{2}$ & 450 & $\mathrm{H}$ \\
\hline F & $5 / 4$ & 386 & $2^{5 \cdot 3 \cdot 5}$ & 480 & $\mathrm{H}$ \\
\hline Fis & $4 / 3$ & 498 & $2^{9}$ & 512 & $\ell$ \\
\hline G & $45 / 32$ & 590 & $2^{2} \cdot 3^{3} \cdot 5$ & 540 & $\mathrm{H}$ \\
\hline Gis & $3 / 2$ & 702 & $2^{6} \cdot 3^{2}$ & 576 & $\mathrm{~h}$ \\
\hline A & $25 / 16$ & 773 & $2^{3 \cdot 3 \cdot 5^{2}}$ & 600 & $\mathrm{H}$ \\
\hline B & $5 / 3$ & 884 & $2^{7 \cdot 5}$ & 640 & $\ell$ \\
\hline H & $225 / 128$ & 977 & $3^{3 \cdot 5}$ & 675 & $\mathrm{H}$ \\
\hline C & $15 / 8$ & 1088 & $2^{4} \cdot 3^{2} \cdot 5$ & 720 & $\mathrm{H}$ \\
\hline \hline
\end{tabular}

Le rapport de fréquences est donné dans la première colonne. La valeur en cents de ce rapport $1200 \cdot \log _{2}(\mathrm{r})$ figure dans la deuxième colonne. Rappelons que le demi-ton du système tempéré vaut 100 cents, ce qui permet des comparaisons faciles. On voit ainsi que le do dièse est de 29 cents inférieur au do dièse du système tempéré (quasiment un sixième de ton). De même les notes altérées (ré dièse, sol dièse, si bémol, à l'exception du fa dièse) sont toutes inférieures à leur équivalent tempéré. Les colonnes suivantes (troisième, quatrième et cinquième colonnes) reprennent les valeurs données par Euler, de la décomposition en facteurs premiers, de la longueur de cordes équivalentes pour ce son construite à partir de la valeur arbitraire d'un do de 384 unités et de l'intervalle formé par la note référencée dans la ligne où il figure et la ligne suivante.

3 Tentamen, Euler 1739. Ch. 9 De genere diatonico-chromatico.

4 Euler remarque que ces intervalles étaient connus de Johann Mattheson (1681-1764), Grosse General Bass Schule, Hamburg, 1731. Cet ouvrage reprend en l'augmentant le premier livre de Mattheson, Exemplarische Organisten Probe, 1719. 
Euler étudie ensuite toutes les transpositions de ce genre sur les différents degrés et les intervalles qui sont ainsi engendrés. Il constate alors que les transpositions engendrent parfois des intervalles nouveaux. Le ton majeur qui vaut 9/8 entre do et ré, ou entre fa et sol vaut un ton augmenté d'un diaschisma (2048/2025) dans le cas de l'intervalle ré dièse, $f a$ ou le cas de l'intervalle si bémol $-d o$. La tierce mineure (6/5) est diminuée d'un comma syntonique $(81 / 80)$ dans trois cas (D:F, Fis:A, B:cs). La quarte $f a-s i$ bémol (675/512) est une quarte juste (4/3) diminuée d'un diaschisma (2048/2025). C'est aussi une quarte augmentée (27/20) d'un comma syntonique sur do dièse (Cis:Fis) et la $(A: d)$, une quarte diminuée (25/18) d'un demi-ton mineur sur ré (D:Gis) et sol (G:cs) et une quarte augmentée (64/45) d'un demi-ton majeur sur ré dièse, $f a$ dièse, si bémol et si bécarre (Dis:A, Fis:c, B:e, $H: f$ ). Toutes ces différences par rapport au tempérament égal donnent à chaque tonalité une couleur différente. Mais ce sont des questions esthétiques qui ne sont pas traitées par Euler, bien qu'il en fût, semble-t-il, parfaitement conscient. Il revient sur la question des consonances du genre diatonico-chromatique au chapitre XI. Au chapitre X, il présente le genre chromatique enharmonique $3^{2} 5^{3}$ et les seize sons du genre $3^{3} 5^{3}$ qui contient l'intervalle de diesis (128/125) de 41 cents proche d'un quart de ton. Il présente même des genres microtonals bien particuliers de 24 sons, le genre $3^{7} 5^{2}$ (Tentamen, 156 ) et le genre $3^{3} 5^{5}$ (Tentamen, 158) qui présentent des intervalles de quart de ton de 51 cents $(16875 / 16384)$ analysé comme un demi-ton mineur (25/24) diminué d'un diaschisma (2048/2025) et des intervalles proches du sixième de ton de 30 cents (3125/3072), analysé comme un demi-ton mineur (24/24) diminué d'un diesis (128/125).

Pour conclure le chapitre, Euler donne la présentation du genre $35^{3} 5^{2} 7$ où pour la première fois intervient le chiffre 7 . On ne sait pas si Euler connaissait la lettre de Leibniz à Golbach à l'époque où il écrivit le Tentamen, bien qu'il cite Leibniz dans le Tentamen, sans toutefois donner de références précises (Euler 1739, p. 163 [Ch. X, \$ 19]. Dans la lettre à Golbach, Leibniz écrit :

Nous, en musique, nous ne comptons pas au delà de cinq, semblables en cela à ces peuples qui, même en arithmétique, n'allaient pas au delà du nombre trois et pour lesquels vaut ce dicton allemand relatif aux personnes simples : il ne sait pas compter après trois [Er kann nicht über drei zahlen]. Car tous les intervalles que nous utilisons proviennent des rapports composés par les rapports existant entre deux des nombres premiers 1, 2, 3 et 5. S'il nous était donné un peu plus de subtilité, nous pourrions aller au nombre premier 7 . Je crois qu'il y a en réalité de telles personnes. C'est pourquoi les anciens musiciens ne repoussaient pas absolument le nombre 7. Mais il y aura peu de personnes pour aller jusqu'aux nombres premiers suivants, 11 et 13. (Leibniz 1988)

La finalité harmonique réside-t-elle dans l'assimilation des nombres premiers de rang supérieur ? Certains y croient. Jacques Chailley, par exemple, dans son Traité historique d'analyse harmonique, explique l'évolution musicale par l'acquisition progressive des sons harmoniques de rang de plus en plus élevés justifiant l'arrivée des accords de $11^{\mathrm{e}}$ et de $13^{\mathrm{e}}$ de dominante. D'autres encore, tels les partisans de l'intonation juste comme Harry Partch, développent des systèmes acoustiques construits sur des rapports fréquentiels faisant intervenir 
certains nombres premiers. Euler n'avait sans doute pas le recul historique suffisant pour juger du rôle des nombres premiers dans l'évolution harmonique. Toujours est-il qu'il franchit le seuil du nombre 5 et donne dans son Tentamen une présentation du genre $33^{3} 5^{2}$. À trois sons près (de rapports $25 / 24,4 / 3$ et $5 / 3$ ), ce système contient tous les sons du genre diatonico-chromatique. Il est toutefois beaucoup plus précis et plus proche des systèmes micro-intervallaires que du tempérament égal. Il compte un intervalle très petit de 8 cents (225/224). Voici la table en notation moderne :

\begin{tabular}{|c|c|c|c|c|c|}
\hline & Fréq. & Cents & Long. & Expr. & Interv. \\
\hline$F$ & 1 & 0 & 4096 & $2^{12}$ & $525 / 512$ \\
\hline Fis $^{*}$ & $525 / 51$ & 43 & 4200 & $2^{3 \cdot 3 \cdot 5^{2} \cdot 7}$ & $36 / 35$ \\
\hline Fis & $135 / 128$ & 92 & 4320 & $2^{3 \cdot 3^{3} \cdot 5}$ & $28 / 27$ \\
\hline$G^{*}$ & $35 / 32$ & 155 & 4480 & $2^{5 \cdot 5 \cdot 7}$ & $36 / 35$ \\
\hline G & $9 / 8$ & 204 & 4608 & $2^{9} \cdot 3^{2}$ & $525 / 512$ \\
\hline Gis $^{*}$ & $4725 / 4096$ & 247 & 4725 & $3^{3.5^{2} \cdot 7}$ & $64 / 63$ \\
\hline Gis & $75 / 64$ & 275 & 4800 & $2^{6} \cdot 3 \cdot 5^{2}$ & $21 / 20$ \\
\hline$A^{*}$ & $315 / 256$ & 359 & 5040 & $2^{4 \cdot 3^{2} \cdot 5 \cdot 7}$ & $64 / 63$ \\
\hline$A$ & $5 / 4$ & 386 & 5120 & $2^{10} \cdot 5$ & $21 / 20$ \\
\hline$B^{*}$ & $21 / 16$ & 471 & 5376 & $2^{8 \cdot 3 \cdot 7}$ & $225 / 224$ \\
\hline$B$ & $675 / 512$ & 478 & 5400 & $2^{3} \cdot 3 \cdot 5^{2}$ & $28 / 27$ \\
\hline$H^{*}$ & $175 / 128$ & 541 & 5600 & $2^{5 \cdot 5^{2.7}}$ & $36 / 35$ \\
\hline$H$ & $45 / 32$ & 590 & 5760 & $2^{7} \cdot 3^{2} \cdot 5$ & $21 / 20$ \\
\hline$C^{*}$ & $189 / 128$ & 675 & 6048 & $2^{5 \cdot 3^{3} \cdot 7}$ & $64 / 63$ \\
\hline$c$ & $3 / 2$ & 702 & 6144 & $2^{11} \cdot 3$ & $525 / 512$ \\
\hline $\operatorname{cis}^{*}$ & $1575 / 1024$ & 745 & 6300 & $2^{2 \cdot} \cdot 3^{2 \cdot} \cdot 5^{2 \cdot 7}$ & $64 / 63$ \\
\hline cis & $25 / 16$ & 773 & 6400 & $2^{8 \cdot 5^{2}}$ & $21 / 20$ \\
\hline$d^{*}$ & $105 / 64$ & 857 & 6720 & $2^{6 \cdot 3 \cdot 5 \cdot 7}$ & $36 / 35$ \\
\hline$d$ & $27 / 16$ & 906 & 6912 & $2^{8 \cdot 3^{3}}$ & $28 / 27$ \\
\hline $\operatorname{dis}^{*}$ & $7 / 4$ & 969 & 7168 & $2^{10.7}$ & $225 / 224$ \\
\hline dis & $225 / 128$ & 977 & 7200 & $2^{5 \cdot 3^{2} \cdot 5^{2}}$ & $21 / 20$ \\
\hline$e^{*}$ & $945 / 512$ & 1061 & 7560 & $2^{3} \cdot 3^{3} \cdot 5 \cdot 7$ & $64 / 63$ \\
\hline e & $15 / 8$ & 1088 & 7680 & $2^{9 \cdot 3 \cdot 5}$ & $21 / 20$ \\
\hline$f^{*}$ & $63 / 32$ & 1173 & 8064 & $2^{7} \cdot 3^{2 \cdot 7}$ & $64 / 63$ \\
\hline$f$ & 2 & 1200 & 8192 & $2^{13}$ & - \\
\hline
\end{tabular}

Au delà de la nouveauté posée par ce système, on retiendra notamment le principe d'engendrement des genres à partir d'une formule unique qui distribue dans l'espace toutes les fréquences autorisées et préfigure une esquisse de classification des systèmes acoustiques. Ces techniques seront reprises, amplifiées et améliorées par les auteurs contemporains, les compositeurs et théoriciens américains, mais aussi par certains mathématiciens comme Yves Hellegouarch qui ont cherché à analyser les gammes naturelles. 
Un autre aspect traité par Euler est ce que l'on pourrait appeler le pouvoir adaptatif de l'oreille. Partant de l'accord de septième $(\mathrm{G}, \mathrm{H}, \mathrm{d}, \mathrm{f})$ exprimé en nombres $(36,45,54,64)$, il remarque que cet accord paraît numériquement compliqué, car le plus petit commun multiple vaut 8640. L'exposant de cet accord est donc très grand. Mais ce qui est perçu est parfois proche et différent de la réalité numérique.

Il est donc suffisamment prouvé, que la proportion aperçue par les sens est
souvent différente de celle qui subsiste actuellement entre les sons. Toutes
les fois que cela arrive, la proportion aperçue est plus simple que la réelle,
et la différence est si petite qu'elle échappe à la perception : l'organe de
l'ouïe est accoutumée de prendre pour une proportion simple, toutes les
proportions qui n'en diffèrent que fort peu, de sorte que la différence soit
quasi imperceptible. (Euler 1764, p. 169)

Euler pense que lorsqu'elle entend un accord de septième, une oreille parfaite comprend les proportions que renferment les nombres que l'accord représente, mais une oreille moins parfaite a tendance à substituer d'autres nombres. C'est pourquoi lorsque l'on entend un accord de septième, certains se réfèrent à $(36,45,54,64)$ alors que d'autres se rattachent à $(36,45,54,63)$ en substituant le nombre 63 à 64 , formant ainsi un rapport plus simple qui se réduit à $(4,5,6$, 7) dont l'exposant vaut 6o. Ainsi, on voit réapparaître le nombre 7 qui justifie

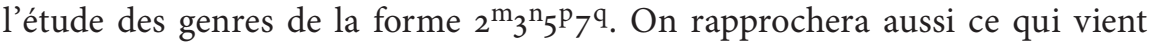
d'être dit des conceptions musicales de Leonhard Euler de l'argument de JeanBaptiste Benedetti en faveur du tempérament égal. Car si le pouvoir adaptatif de l'oreille est réel, le passage invoqué ne devrait pas poser de problème puisque l'écart d'un comma syntonique pourrait être assimilé par l'oreille à un simple unisson. Il aurait été intéressant d'avoir l'avis de notre mathématicien sur cette question, mais - et peut-être à juste titre - Euler ne situe pas sa théorie des genres dans l'ensemble des questions tournant autour du tempérament égal. Il semble que cette théorie des genres dépasse des questions du tempérament, d'une part, parce qu'il donne un principe générateur des genres et des systèmes acoustiques, et d'autre part, parce qu'il situe sa théorie comme une arithmétique cachée dans la musique et les questions de perception. Dans l'article de 1766, Euler voit dans le système des 24 sons le véritable caractère de la musique moderne. Après avoir décrit quelques accords avec des sons étrangers, il remarque :

Mais comme il n'y a aucun doute, que tous ces accords produiraient un meilleur effet, si l'on pouvait exprimer exactement sur les instruments les sons étrangers qui y entrent, la musique pourrait aussi de ce côté être portée à un haut degré de perfection, si l'on trouvait le moyen de doubler le nombre de tons sur les clavecins. Comme les douze tons ordinaires d'une octave sont compris dans cet exposant $2^{\mathrm{m}_{3}} 3^{2} 5^{2}$, dont tous les diviseurs fournissent ces douze tons, il faudrait alors employer cet exposant $2^{\mathrm{m}} 3^{3} 5^{2} 7$, qui fournit 24 tons dans chaque octave, c'est-à-dire douze nouveaux pour représenter exactement les tons, que je nomme étrangers. (Euler 1766, p. 198-199) 
L'intégration de ces tons étrangers est u ne véritable invitation à la microintervallité. Nous voudrions maintenant examiner brièvement ce que sont devenus, au fil des années, les genres ou plus généralement la réorganisation des systèmes acoustiques, indépendamment de la question du tempérament égal.

\section{LA RÉCEPTION ET L'HÉRITAGE DES THÉORIES D'EULER}

Il ne faut pas croire que les genres d'Euler n'ont pas eu de descendance. Au $\mathrm{XIX}^{\mathrm{e}}$ siècle, les recherches sur les réseaux harmoniques se poursuivent dans l'esprit d'Euler avec, entre autres, Hugo Riemann, Ottokar Hostinsky et Arthur von Oettingen', mais elles prennent un nouvel essor au XX ${ }^{\mathrm{e}}$ siècle. C'est le physicien Adriaan Fokker (1889-1972), bien connu pour avoir établi dans sa thèse l'équation qui porte son nom (Équation de Fokker-Planck), et cousin de l'ingénieur spécialiste des questions aéronautiques Anthony Fokker, qui réintroduit les genres musicaux. Non pas comme l'avait fait Euler dans le système classique des douze sons, mais dans un système plus compliqué de 31 sons par octave. Ce système avait déjà été envisagé par Christiaan Huygens (1629-1695) au XVII siècle. Dans la lettre sur le cycle harmonique, Huyghens (Huyghens 1691a) avait montré que l'on pouvait approcher le tempérament mésotonique basé sur des tierces justes par un tempérament égal à 31 degrés. À la même époque, Nicola Vicentino et Fabio Colonna ont envisagé de construire des instruments pour ce tempérament, dont l'archicembalo de Vicentino est resté célèbre. Mais la première publication qui donne le calcul des longueurs des cordes de ce tempérament date de 1666 (Lemme Rossi, Sistema musico).

Il y a dans le troisième livre de la musique de Salinas ${ }^{6}$ un chapitre entier sur le sujet, dont l'inscription est, De prava constitutione cujusdam instrumenti, quod in Italia citra quadraginta annos fabricare coeptum est, in quo reperitur omnis tonus in partes quinque divisus. Il dit que cet instrument était nommé Archicymbalum, qu'il était incerti authoris, que certains musiciens fort habiles l'avaient en grande estime ; et particulièrement de ce qu'il avait tous les intervalles, et toutes les consonances (comme ils croient dit-il) en dessus et en dessous, et qu’après une certaine période on y revenait au même son, ou équivalent, d'où on était parti. Que l'octave y était divisée en 31 parties égales, qu'ils appelaient diesis, desquelles le ton en devait contenir 5 , le grand semiton 3 , le petit 2, la tierce majeure 10, la tierce mineure 8 , la quarte 13 , la quinte 18 , la sixte mineure 21 , la sixte majeure 23 . Mais il ajoute, qu'ayant essayé d'accorder un instrument de cette façon, il a rendu un son fort désagréable, et qui offensait extrêmement les oreilles de tous les assistants. De sorte qu'il conclut qu'un tel accord s'éloigne de toute raison harmonique, soit qu'on l'examine sur le pied des consonances justes, ou de celles du tempérament. (Huyghens 1691a, p. 80)

Le système trentunisonique est donc divisé en 31 diesis. Comme le ton est divisé en cinq parties égales, qui définissent le grand demi-ton et le petit demi-ton, le système permet de distinguer les dièses des bémols, et la notion

5 On pourra consulter Riemann 188o, Hostinsky 1879 et Oettingen 1866.

6 Huyghens fait référence au livre de Francisco Salinas. 1577. De musica libri III. Salamanca. 
d'enharmonie (qui fait que l'on confond un do dièse et un ré bémol) n’a pas lieu ici. Voici comment Fokker présente la construction de ce tempérament :

Mais de quoi s'agit-il au fond ? Ne faisons-nous pas la musique avec quelques intervalles fondamentaux : l'octave (2:1), la quinte (3:2), la tierce majeure (5:4), et la septième harmonique (7:4) ? En disant que nous les additionnons ou soustrayons, nous multiplions les fractions correspondantes et ce que nous additionnons et soustrayons en vérité, ce sont les logarithmes de ces fractions. Voici les logarithmes approximatifs de l'octave $\mathrm{O}=$ $\log 2=0,30103000$, de la septième $S=\log 7 / 4=0,24303805$, de la quinte $\mathrm{Q}=\log _{3} / 2=0,17609125$, de la tierce $\mathrm{T}=\log 5 / 4=0,09691001$. Nous avons affaire à quatre nombres irrationnels. Nous désirons reproduire sur nos instruments les intervalles comme multiples d'un intervalle élémentaire, qui se trouvera entre toutes les paires de touches voisines. C'est-à-dire que nous désirons connaître un diviseur commun de ces intervalles. Or, les logarithmes, en tant que nombres irrationnels, n'ont point de diviseur commun. Cela est impossible. Il faut donc se contenter d'une approximation plus ou moins passable. Voici le procédé. Le plus petit des nombres $\mathrm{T}$ sera soustrait des autres autant de fois que possible, afin de laisser des restes aussi petits que possible, fussent-ils négatifs. Après quoi, ce sont ces restes qu'il s'agira de représenter le mieux possible par des multiples d'un diviseur commun. On obtient ainsi : $\mathrm{O}=3 \mathrm{~T}+\mathrm{R}_{1}$, où $\mathrm{R}_{1}=0,0103=1 \times 0,0103$, $\mathrm{S}=3 \mathrm{~T}+\mathrm{R}_{2}, \mathrm{R}_{2}=-0,0477=-5 \times 0,0095, \mathrm{Q}=2 \mathrm{~T}+\mathrm{R}_{3}, \mathrm{R}_{3}=-0,0177=-2 \times 0,0088, \mathrm{~T}=\mathrm{R}_{4}$, $\mathrm{R}_{4}=0,0969=10 \times 0,0097$. Par ce calcul rapide, nous trouvons que les restes se prêtent à une approximation de $1,-5,-2$, 10 fois un diviseur à peu près constant. À l'aide de ces nombres, nous trouvons un tempérament où les intervalles seront approximativement définis par les nombres O:S:Q:T (=) 31:25:18:10. Nous retrouvons le tempérament de Huygens avec trente-et-un cinquièmes de tons dans l'octave. (Fokker 1947, p. 15)

La construction de ce tempérament est donc empirique. Fokker regrette de ne pas avoir de méthode mathématique lui permettant d'approcher simultanément trois ou quatre nombres irrationnels. Il donne toutefois une autre justification du tempérament trentunisonique en reprenant les idées de Bosanquet?. Sachant que l'octave comprend cinq secondes majeures $(\mathrm{s}+\mathrm{f})$ et deux secondes mineures (f), Fokker écrit l'octave comme l'équation $\mathrm{O}=5 \mathrm{~s}+7 \mathrm{f}$. Puis la quinte $\mathrm{Q}=3 \mathrm{~s}+4 \mathrm{f}$, et la tierce majeure $\mathrm{T}=2 \mathrm{~s}+2 \mathrm{f}$. Il remarque que, dans le tempérament des anciens, $\mathrm{s}$ est supérieur à $\mathrm{f}$, alors que dans le tempérament des classiques, c'est l'inverse : $s$ est inférieur à $\mathrm{f}$. Il en déduit que les anciens ont des approximations à $17(\mathrm{~s}=2, \mathrm{f}=1), 29(\mathrm{~s}=3, \mathrm{f}=2), 41(\mathrm{~s}=4, \mathrm{f}=3)$ et $53(\mathrm{~s}=5, \mathrm{f}=4)$ degrés par octave, et les classiques approchent l'octave avec $19(\mathrm{~s}=1, \mathrm{f}=2), 31(\mathrm{~s}=2, \mathrm{f}=3)$ ou $43(s=3, f=4)$ degrés. Pour représenter ces tempéraments, Fokker se place dans un espace à trois dimensions, figurant les quintes, les tierces et les septièmes, autrement dit les puissances (n, p, q) des exposants d'Euler $3^{\mathrm{n}} 5^{\mathrm{p}} 7^{\mathrm{q}}$ (indépendantes de la puissance $\mathrm{m}$ de $2^{\mathrm{m}}$ qui est déterminée par l'appartenance du rapport acoustique à l'intervalle de l'octave $[1,2])$. 
On peut suivre la même voie si l'on se sert du réseau harmonique à trois dimensions. Persistons dans l'identification de deux tons différant d'un comma. D'un point arbitraire du réseau, d'un son quelconque, on tracera une arête au point où se trouve le second son, qui sera considéré comme homophonique. Ce sera un vecteur d'homophonie. Les coordonnées de ce point seront quatre quintes, et une sous-tierce, c'est-à-dire $(4,-1,0)$. Plus subtile encore est la différence entre les deux demi-tons majeurs $15: 16$ et $14: 15$, ou, ce qui revient au même, la distinction de 224 et 225 . Alors on identifiera 224 et $225,2^{5} .7$ et $3^{2} .5^{2}$, et on tracera dans le réseau un second vecteur d'homophonie, de l'origine au point qui diffère de deux quintes, de deux tierces, et d'une sous-septième, de coordonnées $(2,2,-1)$. En dernier lieu on remarquera que trois septièmes suivies d'une quinte nous portent à un son presque identique au son de départ $\left(3 \times 7 \times 7 \times 7=1029,2^{10}=1024\right)$. Nous identifierons ces deux sons. Dans le réseau harmonique cette paire de sons nous fournit un troisième vecteur d'homophonie, aux coordonnées $(1,0,3)$. (Fokker 1947, p. 11)

Dans le réseau décrit par Fokker, les commas au sens de Yves Hellegouarch (1983) $81 / 80=2^{-4} 3^{4} 5^{-1}, 225 / 224=2^{-5} 3^{2} 5^{2} 7^{-1}$ et $1029 / 1024=2^{-10} \cdot 3 \cdot 7^{3}$ sont assimilés à des unités. Ils définissent des vecteurs d'homophonie dont le volume (le déterminant de la matrice des vecteurs) détermine le nombre de degrés du tempérament :

$$
\left|\begin{array}{ccc}
4 & 2 & 1 \\
-1 & 2 & 0 \\
0 & -1 & 3
\end{array}\right|=31
$$

L'intégration de la septième harmonique impose de sortir du tempérament ordinaire à douze sons. "Il manquait, dit Fokker, une notation convenable, il manquait un système, et, surtout, le tempérament égal à douze demi-tons excluait toute possibilité de reproduire tant soit peu fidèlement la septième parfaite avec les instruments à touches fixes. » (Fokker 1951, p. 2) Tout cela Fokker le trouve dans le tempérament à 31 sons. Il invente une nouvelle notation, fait construire un orgue pour expérimenter ce tempérament et passe commande aux compositeurs hollandais. Sous son nom ou sous son pseudonyme (Arie de Klein), Fokker compose des petites pièces pour l'orgue trentunisonique qui utilisent les genres d'Euler avec septième harmonique : Canzone $\left(3^{2} \cdot 7\right)$, Preludium chromaticum (3.5.7), Improvisando $\left(5^{2} \cdot 7\right)$, Inventio $\left(5 \cdot 7^{2}\right)$, Capriccio $\left(7^{3}\right)$. En 1957, il compose un recueil de cinq pièces In generibus Leonhardi Euleri : Toccata $\left(3^{2} \cdot 5 \cdot 7\right)$, Barcarole e toccata $\left(3 \cdot 5^{2} \cdot 7\right)$, Il sogno di Giacomo $\left(3 \cdot 5 \cdot 7^{2}\right)$, Mordente e toccata $\left(5^{3} \cdot 7^{2}\right)$ et Mosaico e toccata $\left(5^{2} \cdot 7^{3}\right)$. Il influence aussi les compositeurs hollandais qui, comme Jan van Dijk, se mettent à expérimenter les genres d'EulerFokker. Douze pièces de van Dijk composées en 1951 ont été regroupées sous le titre Musica per organo trentunisono ; toutes utilisent les genres d'Euler. Une même pièce est parfois construite sur plusieurs genres : Prélude et fugue pour hautbois et orgue $\left(5 \cdot 3^{4}, 3 \cdot 5 \cdot 7\right.$ et $\left.3^{4} \cdot 7\right)$, Fugue pour violon, alto et orgue $\left(3^{3} \cdot 7^{3}, 3^{2} \cdot 7^{3}\right.$, $\left.3 \cdot 7^{4}, 3^{4} \cdot 7\right)$. Canon pour orgue seul $\left(7^{5}\right)$, Fugue pour flûte, deux hautbois et orgue $\left(3^{2} \cdot 5^{2} \cdot 7\right)$, Toccata pour orgue seul $\left(3^{2} \cdot 5 \cdot 7^{2}\right)$, Fugue pour hautbois, violon et orgue $\left(3 \cdot 5^{3} \cdot 7\right)$, Prélude et fugue pour orgue seul $\left(3 \cdot 5^{2} \cdot 7^{2}\right)$, Corrente alla fuga pour orgue 
seul (3.5.73), Capriccio pour flûte en sol et orgue ( $\left.5^{4} \cdot 7\right)$, Fugue pour flûte en sol et orgue $\left(5^{3} \cdot 7^{2}\right)$, Duettino e Ciaconna pour deux flûtes et orgue $\left(5^{2} \cdot 7^{3}, 5 \cdot 7^{4}\right)$, Basso solo pour pédalier $\left(7^{5}\right)$.

Aux États-Unis, les adeptes de l'intonation juste ${ }^{8}$ prônent un retour à l'emploi d'intervalles dont les rapports de fréquence sont des nombres simples. Les systèmes acoustiques sont construits dans le même esprit que les genres musicaux d'Euler. L'intonation juste est, selon la définition de Harry Partch, « un système dans lequel la construction d'intervalles - et d'échelles - est fondée sur le critère auditif et par conséquent est un système et une procédure limités à de petits nombres fractionnaires; l'intervalle initial de l'intonation juste est 2/1. Il s'ensuit une abondance d'intervalles musicaux basés sur des relations tonales impliquant des petits nombres. L'intonation juste est le terme générique pour décrire cette procédure. » (Partch 1974, p. 71)

La procédure de construction que propose Partch est voisine de celle des genres musicaux. Elle consiste à remplir les cases d'un losange (tonality diamond) en attribuant à chaque case une fraction numérique qui reçoit un nombre impair au numérateur ou au dénominateur selon que le remplissage se fait le long d'une diagonale ou le long de la diagonale perpendiculaire. Pour le nombre impair 5 , on place dans un losange représentant $3 \times 3$ cases, les nombres 1,3 et 5 dans les numérateurs et les dénominateurs des fractions. On divise ensuite chaque nombre obtenu par une puissance de 2 de façon à ramener le nombre dans une octave $[1,2]$. En réordonnant, on obtient pour la limite 5 , le système acoustique $\{1,6 / 5,5 / 4,4 / 3,3 / 2,8 / 5,5 / 3,2\}$ dont la parenté avec les genres d'Euler est patente. Le nombre de degrés du genre est variable et dépend de la limite choisie. Pour une limite 5, on a 7 degrés par octave, 13 degrés en limite 7, 19 degrés en limite 9, 29 degrés en limite 11 et 41 degrés en limite 13. Cette procédure ne permet pas de retrouver le tempérament à 31 degrés de Huygens-Fokker, mais offre une série de nouveaux réseaux qui seront exploités par Partch et les compositeurs américains. La méthode d'Ervin Wilson (appelée Combination Product Sets) s'apparente à la méthode de Partch. ${ }^{9}$

Dans des œuvres comme The Well-Tuned Piano de La Monte Young composé en 1964, ou les Études dissonantes (1998) de Waren Burt, l'accord des instruments joue un rôle central dans la composition. À la manière de Fokker, le compositeur Ben Johnston construit des tempéraments en prélevant des suites de sons dans des réseaux harmoniques de 2 à 10 dimensions. The Song of Innocence (1975) pour soprano et ensemble instrumental et Two Sonnets of Shakespeare (1979) sont construits sur le réseau bidimensionnel 3·11. Johnston emploie le genre 3.5.7 dans One Man (1967) pour trombone solo, Rose (1971) pour chœur, Mass (1972) pour chœur, dix trombones, contrebasse et percussions et dans le Quatrième quatuor à cordes (1973). Dans Diversion (1979) pour onze instruments et dans le Sixième quatuor à cordes, il explore le genre $3 \cdot 5 \cdot 7 \cdot 11$. Duo pour deux violons (1978) et le Cinquième quatuor à cordes (1979) sont construits sur

8 L'intonation a aussi des partisans en dehors des États-Unis. Par exemple, en France, Jacques Dudon et l'Atelier d'Exploration Harmonique, voir http://aeh.free.fr/.

9 Sur ces questions, voir le livre de Bob Gilmore (1998), ainsi que notre Dictionnaire des musiques microtonales (Jedrzejewski 2003). 


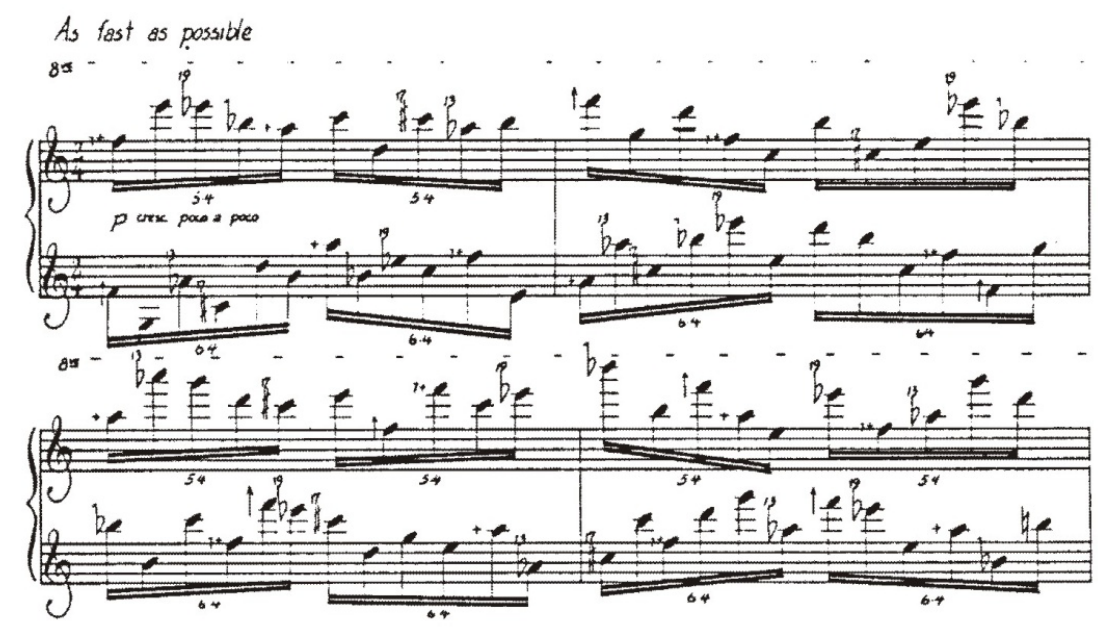

Figure 4. Ben Johnston, Suite microtonale pour piano (1978). (c) Smith Publications Baltimore, Maryland 21207 USA Reproduit avec autorisation.

le genre $3 \cdot 5 \cdot 7 \cdot 11 \cdot 13$. La Suite microtonale pour piano (1978) et les Twelve Partials (1980) pour flûte et piano microtonal emploient le genre $3 \cdot 5 \cdot 7 \cdot 11 \cdot 13 \cdot 17 \cdot 19$. Le Neuvième quatuor à cordes écrit en 1987 explore les ressources d'un réseau à dix dimensions $3 \cdot 5 \cdot 7 \cdot 11 \cdot$ hotmail.com13 $17 \cdot 19 \cdot 23 \cdot 29 \cdot 31$. Ces quelques exemples montrent l'intérêt des théories musicales d'Euler pour la musique contemporaine.

Avec le Tentamen, non seulement Euler donne un modèle mathématique du plaisir musical à travers le degré de suavité ou d'agrément harmonique, mais il le fonde sur le plan physio-psychologique et acoustique en reprenant l'idée évoquée dans la lettre de Leibniz à Goldbach sur la congruence des chocs. Bien qu'il ne traite pas de la question du tempérament égal, il n'en reste pas moins que sa théorie des genres musicaux est la première formulation systématique cohérente de construction de systèmes d'accord des instruments. Mais ce qui est réellement nouveau dans la démarche d'Euler est, non pas de donner une forme mathématique aux rapports acoustiques comme le faisaient les pythagoriciens, mais de formaliser les lois musicales harmoniques dans leur dimension sensible jusqu'à ce quelles ont de plus suaves.

Ce n'est pas uniquement dans l'objet qu'il faut chercher la cause pour laquelle nous le trouvons agréable ou désagréable; il faut aussi avoir égard aux sens qui en représentent l'image à l'esprit et surtout au jugement que l'esprit se forme de cette image. (Euler 1739, p. 21 [Ch. 2])

Le modèle doit donc prendre en considération «l'opinion de ceux qui possèdent un excellent goût et beaucoup de jugement » (Euler 1739, p. 21 [Ch. 2]). Euler réintroduit le sujet dans l'objet musical. Cette formalisation sensible témoigne d'une attitude d'émancipation musicale que l'on retrouve deux siècles plus tard chez les adeptes de l'intonation juste et des univers micro-intervallaires, qui, comme Harry Partch et Erv Wilson, cherchent à construire et à composer avec 
la complexité de nouveaux systèmes acoustiques. Ainsi se dessine une filiation que traduit ce geste commun qui est de donner un sens à la consistance et à la complétion des réseaux harmoniques.

\section{RÉFÉRENCES}

Benedetti, Jean-Baptiste. 1585. Diversarum speculationum mathematicarum, et physicarum liber : quarum seriem sequens pagina indicabit, disponible sur le site "European Cultural Heritage Online ", du Max Planck Institute : http://echo.mpiwg-berlin.mpg.de/.

Bosanquet, R.H.M. 1875. "The Theory of the Division of the Octave, and the Practical Treatment of the Musical Systems thus Obtained », Proc. Roy. Soc. London $23, \mathrm{n}^{\circ} 390$.

Euler, Leonhard. 1739. Tentamen novae theoriae musicae ex certissimis harmoniae principiis dilucide expositae, St. Petersbourg. Traduction française "Essai d'une nouvelle théorie sur la musique ", Euvres complètes vol. 5, 1839, I-VII, 1-215. Publié aussi dans Musique mathématique. Paris : Librairie Scientifique et Philosophique, 1865.

-1764. Conjecture sur la raison de quelques dissonances généralement reçues dans la musique, Mémoires de l'Académie des sciences de Berlin, 165-173.

—.1766. Du véritable caractère de la musique, Mémoires de l'Académie des sciences de Berlin, 174-199.

.1774. De harmoniae veris pincipiis perspeculum musicum repraesentatis, Novi commentarii Academiae scientiarum Petropolitanae, $\mathrm{n}^{\circ} 18: 330-353$.

Fétis, François-Joseph. 1840. «Esquisse de l'histoire de l'harmonie considérée comme art et comme science systématique ». Revue et Gazette musicale de Paris $\mathrm{n}^{\circ} 52: 447-452$.

Fokker, Adriaan. 1947. "Les mathématiques et la musique ». Recherches musicales. Théoriques et pratiques. La Haye: Archives du Musée Teyler $n^{\circ} 10$ : 1-31.

-1955. Equal Temperament and the Thirty-one-keyed Organ. Document de 6 pages.

_.1955-1966. "Simon Stevin's Views on Music », introduction à Vande spiegheling der singconst ("On the Theory of the Art of Singing »). Principal Works of Simon Stevin 5, sous la dir. de Adriaan D. Fokker, 413-464. Amsterdam : C. V. Swets \& Zeitlinger.

1969. «Unison Vectors and Periodicity Blocks in the Three-Dimensional $(3-5-7)$ Harmonic Lattice of Notes ». Koninklijke Nederlandse Akademie van Wetenschappen. Amsterdam, Actes du colloque de février 1969, Série B 72 (3).

—.1975. New Music With 31 Notes, traduit par Leigh Gerdine. Bonn : Verlag für Systematische Musikwissenschaft. Orpheus - Series of Monographs on Basic Questions in Music, vol. 5.

Fokker, Adriaan et Jan van Dijk. 1951. «Expériences musicales avec les genres musicaux de Leonhard Euler contenant la septième harmonique, avec des 
compositions ». Recherches musicales. Théoriques et pratiques. La Haye : Teyler Museum Archives, $\mathrm{n}^{\circ}$ 10, Martinus Nijhoff.

Gilmore, Bob. 1998. Harry Partch, A Biography. New Haven : Yale University Press.

Hellegouarch, Yves. 1992. "L'essai d'une nouvelle théorie de la musique de Leonhard Euler ». Destins de l'art, desseins de la science, Actes du colloque de Caen, 1986, IREM de Basse Normandie.

_. 1987. "À la recherche de l'arithmétique qui se cache dans la musique ». Gazette des mathématiciens, SMF n $33: 71-80$.

_. 1983. "Gammes naturelles ». Musique et mathématiques, Publication de l'APMEP, $\mathrm{n}^{\circ}$ 53. Autre version dans la Gazette des mathématiciens, $\mathrm{n}^{\circ} 81$ (1999) 25-39 et 82 (1999) :13-26.

Hostinsky, Ottokar. 1879. Die Lehre von den musikalischen Klängen : ein Beitrag zur aesthetischen Begründung der Harmonielehre. Prague : H. Domenicus. Huygens, Christian. 1691. Le cycle harmonique. Rotterdam 1691. Réed. par Rasch. 1988. Utrecht: The Diapason Press.

- 1691a. Lettre de Mr. Huygens à l'Auteur touchant le Cycle Harmonique, Histoire des Ouvrages des Sçavans (Henri Basnage de, Beauval). Rotterdam, Octobre 1691, 78-88.

—.1724. "Novus cyclus harmonicus ». Opera varia, tome 20, Leyden. Réed. par Rasch. 1988. Utrecht: The Diapason Press.

Jedrzejewski, Franck. 2002. Mathématiques des systèmes acoustiques, Tempéraments et modèles contemporains. Paris : L'Harmattan.

_.2003. Dictionnaire des musiques microtonales. Paris : L'Harmattan.

. 2006. Mathematical Theory of Music. Sampzon : Éditions IRCAM/Delatour France.

Johnston, Ben. 1976-1977. « Rational Structure In Music». American Society of University Composers Proceedings $\mathrm{n}^{\mathrm{os}} 1-2$ : 102-118. Reprise dans One/one $2, \mathrm{n}^{\circ} 3,1986$, et $2, \mathrm{n}^{\circ} 4,1986$.

. 2006. 'Maximum Clarity' and Other Writings on Music, sous la dir. de Bob Gilmore. Chicago : University of Illinois Press.

Leibniz, Gottfried W. 1988. Correspondance avec Golbach, traduction de Frédéric de Buzon, Philosophie, $\mathrm{n}^{\circ} 59: 10-13$.

. 1863. "Initia mathematica ». Mathematische Schriften, vol. 7. Berlin : Gerhardt.

Oettingen, Arthur von. 1866. Harmoniesystem in dualer Entwiecklung. Dorpat: W. Gläser.

Partch, Harry. 1974. Genesis of a Music: An Account of a Creative Work, its Roots and its Fulfillment. New York : Da Capo Press. Réédition, 1979 ; première édition, 1949.

Rasch, Rudolf. 1987. Adriaan D. Fokker (1887-1972). Utrecht : The Diapason Press.

Riemann, Hugo. 1880. Skizze einer neuen Methode der Harmonielehre. Leipzig. 


\title{
RÉSUMÉ
}

Dans cet article, on montre que le Tentamen de Leonhard Euler (1707-1783) apporte une solution arithmétique et musicale aux problèmes évoqués par Leibniz dans sa lettre à Christian Goldbach du 17 avril 1712, et que, contrairement à une idée largement répandue, les genres musicaux (genus musicum) ont été repris et développés par des théoriciens de la musique et des compositeurs. Tout d'abord par le physicien Adriaan Fokker (1887-1972) qui a sollicité de nombreux compositeurs hollandais, puis par l'école américaine qui, avec Harry Partch (1901-1974), Ervin Wilson (1928) et Ben Johnston (1926), a poursuivi le développement de ces théories dans l'exploration expérimentale de l'intonation juste et des univers non tempérés.

\begin{abstract}
In this article, the author shows that the Tentamen of Leonhard Euler (1707-1783) presents an arithmetic and musical solution to the problems raised by Leibniz in letter to Christian Goldbach of April 17 1712, and that, contrary to a widely held belief, its musical typology (genus musicum) was taken up and developed by subsequent music theorists and composers. First among these was the physician Adriaan Fokker (18871972), who introduced many Dutch composers to Euler's theories; he was followed by the American school, whose members, Harry Partch (1901-1974), Ervin Wilson (b. 1928) and Ben Johnston (b. 1926), have pursued the development of these theories in their experiments in just intonation and the realm of non-tempered scales.
\end{abstract}

